\title{
Effects of 17-DMAG on diffuse large B-cell lymphoma cell apoptosis
}

\author{
JIA-JIA LI，JING-JING ZHANG，XIU WANG and ZI-MIN SUN
}

Department of Hematology, Affiliated Provincial Hospital of Anhui Medical University, Hefei, Anhui 230001, P.R. China

Received May 16, 2016; Accepted July 20, 2017

DOI: $10.3892 /$ etm.2017.4995

\begin{abstract}
. 17-dimethylaminoethylamino-17-demethoxygeldanamycin (17-DMAG) is a water soluble, semisynthetic derivative of endotoxin that has anticancer effects. The aim of the present study was to determine whether 17-DMAG enhances the apoptosis of lymphoma cells in diffuse large B-cell lymphoma. Apoptosis was induced in SU-DHL-4 diffuse large B-cell lymphoma cells treated with 17-DMAG, as evaluated by MTT assay and flow cytometry analysis. Apoptosis-associated protein levels were assessed using western blotting, and the results indicated that B-cell lymphoma 2 (Bcl-2)-associated protein X (Bax) was upregulated, whereas heat shock protein family A member 5 (HSPA5) and Bcl-2 were downregulated. Additionally, staining with 5,5',6,6'-Tetrachloro-1,1',3,3'-tetraethyl-imidacarbocyanine iodide revealed that treatment with 17-DMAG decreased mitochondrial membrane potential in SU-DHL-4 diffuse large B-cell lymphoma cells. These results suggested that 17-DMAG is able to inhibit proliferation in diffuse large B-cell lymphoma cells in a concentration-dependent manner. The underlying mechanism may be that 17-DMAG induces oxidative stress, which inhibits the expression of HSPA5 and Bcl-2 and promotes the expression of Bax, leading to the apoptosis of SU-DHL-4 cells. Taken together, these results indicated that 17-DMAG may be an effective novel agent for the treatment of diffuse large B-cell lymphoma.
\end{abstract}

\section{Introduction}

Diffuse large B-cell lymphoma (DLBCL) is the most commonly diagnosed form of lymphoma in Western and developing countries, accounting for $40 \%$ of non-Hodgkin lymphoma cases among adults (1-3). Conventional chemotherapy and bone marrow transplantations have a curative

Correspondence to: Professor Zi-Min Sun, Department of Hematology, Affiliated Provincial Hospital of Anhui Medical University, 17 Lujiang Road, Hefei, Anhui 230001, P.R. China E-mail: zmsun_VIP@163.com

Key words: 17-dimethylaminoethylamino-17-demethoxygeldanamy cin, diffuse large B-cell lymphoma cells, SU-DHL-4, mitochondrial membrane potential, apoptosis effect and are able to effectively improve the survival rates of patients with DLBCL, with some patients being completely cured (4). Nevertheless, lymphoma relapse and drug resistance are a major challenge in clinical practice (5). In order to improve the therapeutic efficacy and reduce negative impacts of treatment, a combination of techniques are typically used to treat the disease (6).

It has previously been reported that heat shock protein 90 (HSP90) has a notable role in tumor development; it has therefore become a novel target for tumor therapy (7). HSP90 regulates the stability, translation, degradation and function of its client proteins via an adenosine 5'-triphosphate (ATP)-regulated mechanism (7). Unlike other HSPs, HSP90 has 200 cellular target proteins (8-10). HSP90 inhibitors, such as 17-dimethylaminoethylamino-17-demethoxy geldanamycin (17-DMAG), are a group of small molecules that interact and inhibit the activity of HSP90 dimers $(11,12)$. 17-DMAG is able to inhibit the chaperone activity of HSP90 via competing with ATP for its binding site, leading to the misfolding of cellular client proteins, including RAF proto-oncogene serine/threonine-protein kinase, mutant TP53 proteins, proto-oncogene tyrosine-protein kinase $\mathrm{Src}$, receptor tyrosine-protein kinase-2 and the nuclear receptor subfamily 3 group $\mathrm{C}$ member 1 , which results in protein degradation and destabilization via the proteasomal pathway (13). 17-DMAG is able to suppress tumor growth by inhibiting HSP90, which affects the degradation of key proteins involved in multiple signal transduction pathways, thus inhibiting cell proliferation and inducing cell apoptosis (13). The effect of 17-DMAG on DLBCL cells is not completely understood and so there is a requirement for further experimental and clinical studies to determine its value in clinical practice. The purpose of the present study was to determine the effect of 17-DMAG on DLBCL, whether 17-DMAG is able to induce apoptosis in DLBCL cells and the underlying mechanisms responsible.

\section{Materials and methods}

Cell lines and cell culture. The SU-DHL-4 DLBCL cell line (Chinese Academy of Sciences, Shanghai Institute of Life Sciences, Cell Resource Center, Shanghai, China) was maintained in suspension at $37^{\circ} \mathrm{C}$ in an atmosphere containing $5 \% \mathrm{CO}_{2}$. Cells were cultured in Dulbecco's modified Eagle medium (DMEM; Gibco; Thermo Fisher Scientific, Inc., Waltham, MA, USA) supplemented with $10 \%$ fetal bovine 
serum (Hyclone; GE Healthcare Life Sciences, Logan, UT, USA). Cells in the exponential growth phase were then seeded into 96-well plates at a density of $1 \times 10^{4}$ cells/well.

Antibodies and reagents. The HSP90 inhibitor, 17-DMAG, was donated by Dr Hong Young-Soo (South Korean Living Engineering Research Institute, Seoul, South Korea). Rabbit anti-human B-cell lymphoma 2 (Bcl-2, SC-509), rabbit anti-human Bcl-2-associated X protein (Bax, SC-526) and rabbit anti-human HSP70 member 5 (HSPA5, ab21658) antibodies were purchased from Abcam (Cambridge, UK). Rat anti-human $\beta$-actin antibodies (sc-47778) were purchased from Santa Cruz Biotechnology, Inc., (Dallas, TX, USA).

Assessment of cell proliferation by MTT assays. Cell proliferation was analyzed using an MTT assay (M2128, Sigma-Aldrich; Merck KGaA, Darmstadt, Germany). A total of $5 \times 10^{3}$ SU-DHL-4 cells/ $100 \mu 1 /$ well were seeded into 96-well plates, treated with different concentrations of 17-DMAG $(0$, $25,50,100$ and $200 \mu \mathrm{mol} / \mathrm{l}$ ), and cultured for 24,48 and $72 \mathrm{~h}$ in a $5 \% \mathrm{CO}_{2}$ incubator at $37^{\circ} \mathrm{C}$. Each experiment was performed in triplicate. The cells were subsequently incubated with $15 \mu \mathrm{l}$ MTT reagent at $37^{\circ} \mathrm{C}$ for $4 \mathrm{~h}$, and dimethyl sulfoxide was added to dissolve the formazan crystals. An ELI plate reader (Spectra Max M2; Molecular Devices LLC, Sunnyvale, CA, USA) was used to measure the optical density of each well at $570 \mathrm{~nm}$. Finally, the cell survival rate was calculated using the following formula: Cell survival rate $(\%)=($ experimental group A value - zero set A value) / (control group A value - zero set A value) x 100.

Assessment of apoptosis using propidium iodide (PI) staining. The apoptotic rate of the SU-DHL-4 cells was analyzed using PI. Cells in a 6-well plate at a density of $3 \times 10^{5}$ cells/well were treated with different concentrations of 17-DMAG $(0,25,50$, 100 and $200 \mu \mathrm{mmol} / \mathrm{l}$ ) for $24 \mathrm{~h}$ at $37^{\circ} \mathrm{C}$. At the end of the treatment, cells were washed with cold PBS and fixed overnight with alcohol solution at $4^{\circ} \mathrm{C}$. Then the fixed cells were washed twice with PBS, stained with PI solution $\left(5 \mathrm{mg} \mathrm{NaC}_{6} \mathrm{H}_{5} \mathrm{PI}\right.$,

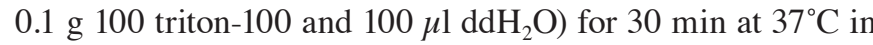
the dark according to the manufacturer's protocol (BBI Life Science Corporation, Shanghai, China). Finally, $\geq 7,000$ events per sample were acquired using a flow cytometer. CellQuest Pro software, version 3.0 (BD Biosciences, Franklin Lakes, NJ, USA) was used to analyze the results of the flow cytometer analysis.

Western blot analysis. Western blot analysis was performed to detect the protein expression levels of Bax, Bcl-2 and HSPA5. Proteins were extracted from the DLBCL cells treated with 17-DMAG and from untreated control cells. The cells were plated in 6-well culture dishes at a density of $6 \times 10^{5}$ cells/well. The harvested cells were then lysed on ice for $30 \mathrm{~min}$ in $100 \mathrm{ml}$ of lysis buffer [120 mmol/1 NaCl, $40 \mathrm{mmol} / \mathrm{l}$ Tris (pH 8), $0.1 \%$ NP40] and centrifuged at $8,600 \times \mathrm{g}$ for $30 \mathrm{~min}$ at $37^{\circ} \mathrm{C}$. The bicinchoninic acid assay method (BCA1-1KT, Sigma Aldrich; Merck KGaA) was used to determine the protein concentration of the samples. Protein samples ( $20 \mu \mathrm{g} / \mathrm{lane})$ were resolved using $10 \%$ SDS-PAGE and blotted onto polyvinylidene difluoride membranes. The membranes were subsequently
Table I. Cell survival rate of SU-DHL-4 cells.

\begin{tabular}{ccc}
\hline & \multicolumn{2}{c}{ Cell survival rate, $\%$} \\
\cline { 2 - 3 } 17-DMAG, $\mu \mathrm{mol} / 1$ & $24 \mathrm{~h}$ treatment & $48 \mathrm{~h}$ treatment \\
\hline 0 & $100.0000 \pm 0.6$ & $100.0000 \pm 0.2^{\mathrm{a}}$ \\
25 & $88.9913 \pm 0.5^{\mathrm{a}}$ & $89.5851 \pm 1.1^{\mathrm{a}}$ \\
50 & $71.5563 \pm 0.2^{\mathrm{a}}$ & $58.1955 \pm 3.0^{\mathrm{a}}$ \\
100 & $62.2241 \pm 0.4^{\mathrm{a}}$ & $55.1494 \pm 2.5^{\mathrm{a}}$ \\
200 & $52.4728 \pm 0.2^{\mathrm{a}}$ & $39.4546 \pm 1.2^{\mathrm{a}}$ \\
\hline
\end{tabular}

17-DMAG, 17-dimethylaminoethylamino-17-demethoxy geldanamycin. ${ }^{\text {aP }}<0.05$ vs. $0 \mu \mathrm{mol} / 1$ 17-DMAG.

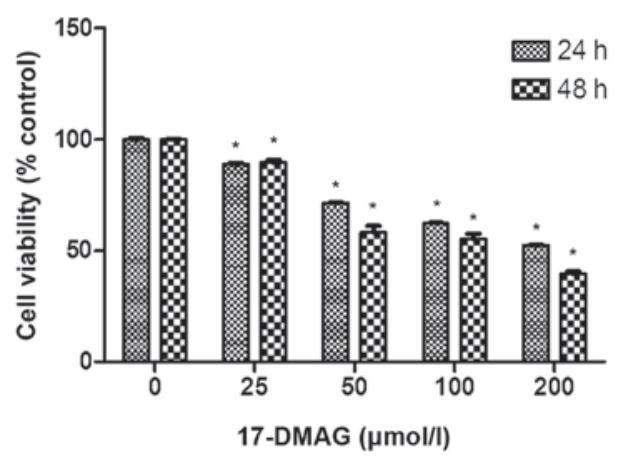

Figure 1. Effects of 17-DMAG on the viability of diffuse large B-cell lymphoma cells. The diffuse large B-cell lymphoma cell line, SU-DHL-4, was treated with 17-DMAG at different concentrations for 24 and $48 \mathrm{~h}$. 17-DMAG, 17-dimethylaminoethylamino-17-demethoxy geldanamycin. ${ }^{*} \mathrm{P}<0.05$ vs. $0 \mu \mathrm{mol} / 1$ 17-DMAG.

incubated with the appropriate primary antibodies against Bax $(1: 1,000), \operatorname{Bcl}-2(1: 1,000)$ and HSPA5 $(1: 1,000)$ to detect each the proteins of interest separately at $4^{\circ} \mathrm{C}$ overnight. $\beta$-actin $(1: 1,000)$ was used as a loading control. After being washed with PBS, the membranes were incubated with peroxidase-conjugated secondary antibodies (ab6728, Abcam, Cambridge, UK) for $2 \mathrm{~h}$ at room temperature. Finally, the detected protein bands were visualized using an Immobilon Western Chemiluminescent HRP Substrate kit (Merck KGaA) and the ImageQuant LAS 4000 software (GE Healthcare Life Sciences, Little Chalfont, UK).

\subsection{5,5',6,6'-Tetrachloro-1,1',3,3'-tetraethylbenzimidazolca-} rbocyanine Iodide (JC-1) staining. Changes in the mitochondrial membrane potential (MMP) were measured using JC-1 staining (Invitrogen; Thermo Fisher Scientific, Inc.) following the manufacturer's protocol. Following treatment with 17-DMAG for $24 \mathrm{~h}$ at $37^{\circ} \mathrm{C}$, SU-DHL-4 cells from each group were incubated with $10 \mu \mathrm{M} \mathrm{JC}-1$ dye at $37^{\circ} \mathrm{C}$ for $30 \mathrm{~min}$. Subsequently, cells were washed twice with PBS and observed under a Zeiss 780 laser scanning confocal microscope (Carl Zeiss SAS, Jena, Germany; magnification, x100), with excitation wavelength at $488 \mathrm{~nm}$ and emission wavelength at 527 and $590 \mathrm{~nm}$. Image-Pro Plus 6.0 software was used to analyse the fluorescence intensity of JC-1 staining. 

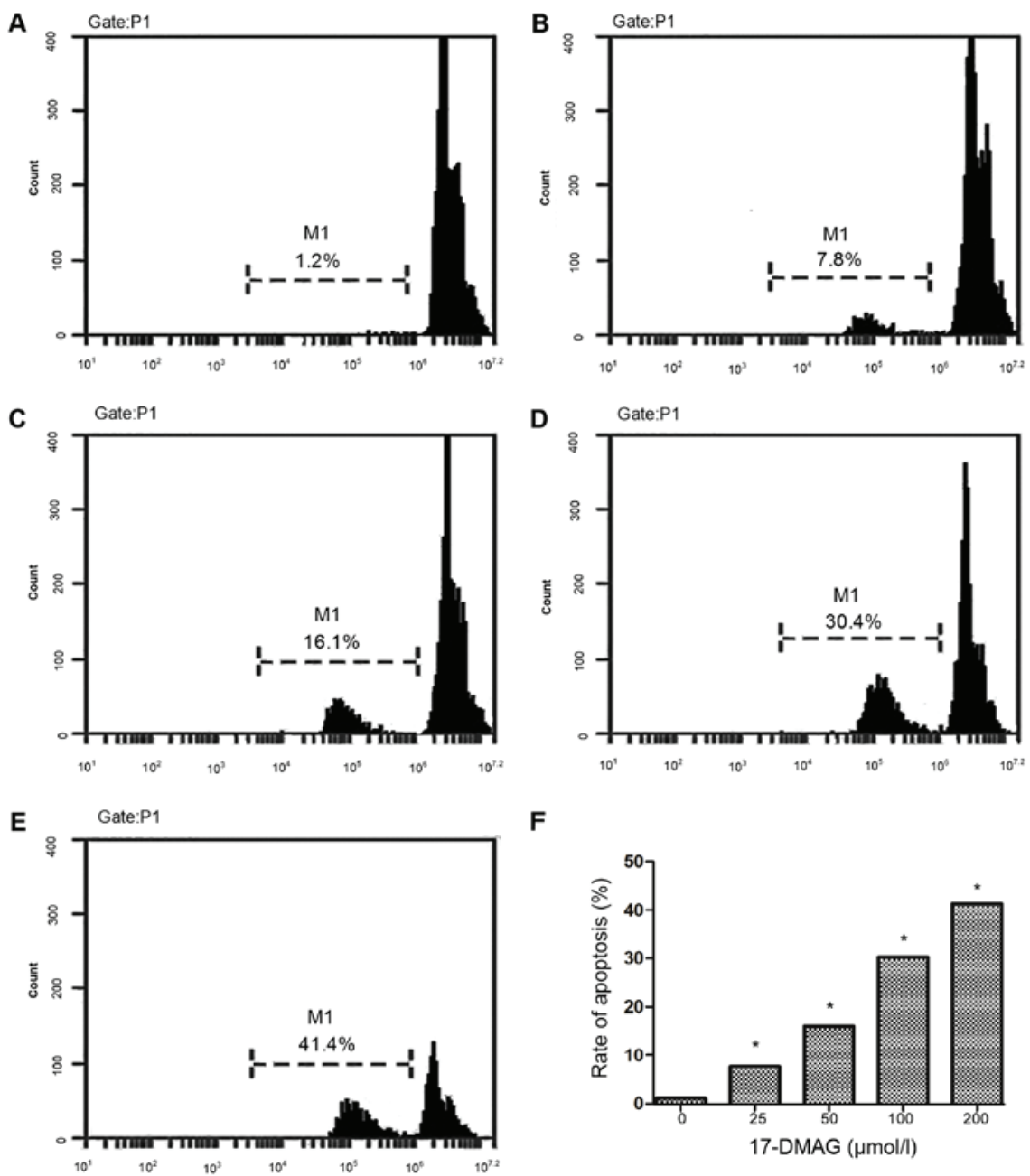

$\mathbf{F}$

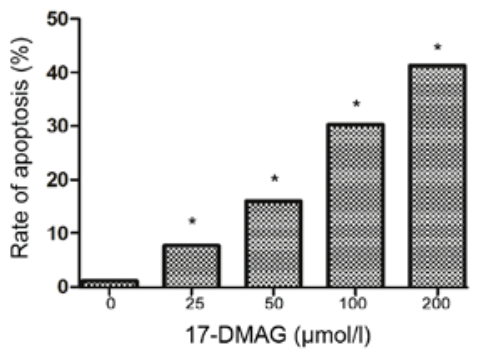

Figure 2. 17-DMAG treatment induces apoptosis in diffuse large B-cell lymphoma cells. Representative flow cytometry profiles for SU-DHL-4 cells treated with (A) 0, (B) 25, (C) 50, (D) 100 and (E) $200 \mu \mathrm{mol} / 1$ 17-DMAG for $24 \mathrm{~h}$. (F) Quantification of the rate of apoptosis. "P<0.05 vs. $0 \mu \mathrm{mol} / 1.17-\mathrm{DMAG}$, 17-dimethylaminoethylamino-17-demethoxy geldanamycin.

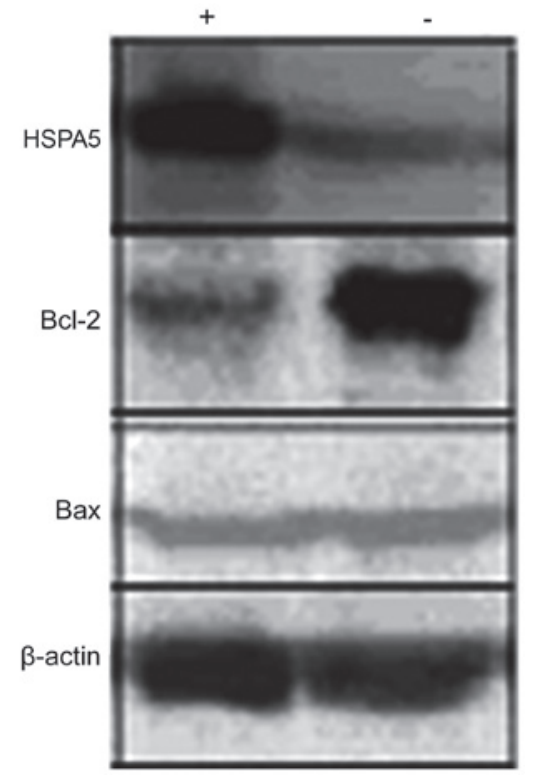

Figure 3. Western blot analysis of apoptosis-related proteins with $\beta$-actin as an equal loading control. HSPA5, heat shock protein family A member 5; Bcl-2, B-cell lymphoma-2; Bax, Bcl-2-associated X protein; +, treatment with 17-DMAG; -, no treatment with 17-DMAG; 17-DMAG, 17-dimethylaminoethylamino-17-demethoxy geldanamycin.
Statistical analysis. All statistical analyses were performed using one-way analysis of variance with least significant difference test on SPSS 18.0 software (SPSS, Inc., Chicago, IL, USA). All results were expressed as the mean \pm standard deviation. $\mathrm{P}<0.05$ was considered to indicate a statistically significant difference.

\section{Results}

17-DMAG suppresses the proliferation of DLBCL cells. The anti-proliferative activity of 17-DMAG was evaluated in the DLBCL cell line, SU-DHL-4. Cells were treated with different concentrations of 17-DMAG for $48 \mathrm{~h}$, and then cell viability was analyzed using an MTT assay. The results revealed that 17-DMAG inhibited the proliferation of SU-DHL-4 in a dose-dependent manner (Table I; $\mathrm{P}<0.05$ vs. $0 \mu \mathrm{mol} / \mathrm{l}$.). The proliferation of SU-DHL-4 cells was also decreased in a time-dependent manner by 17-DMAG treatment (Fig. 1 and Table I; $\mathrm{P}<0.05$ vs. 0 h).

17-DMAG treatment induces apoptosis in DLBCL cells. The effect of 17-DMAG on apoptosis in SU-DHL-4 cells was evaluated to further characterize its antitumor activity. Cells were 

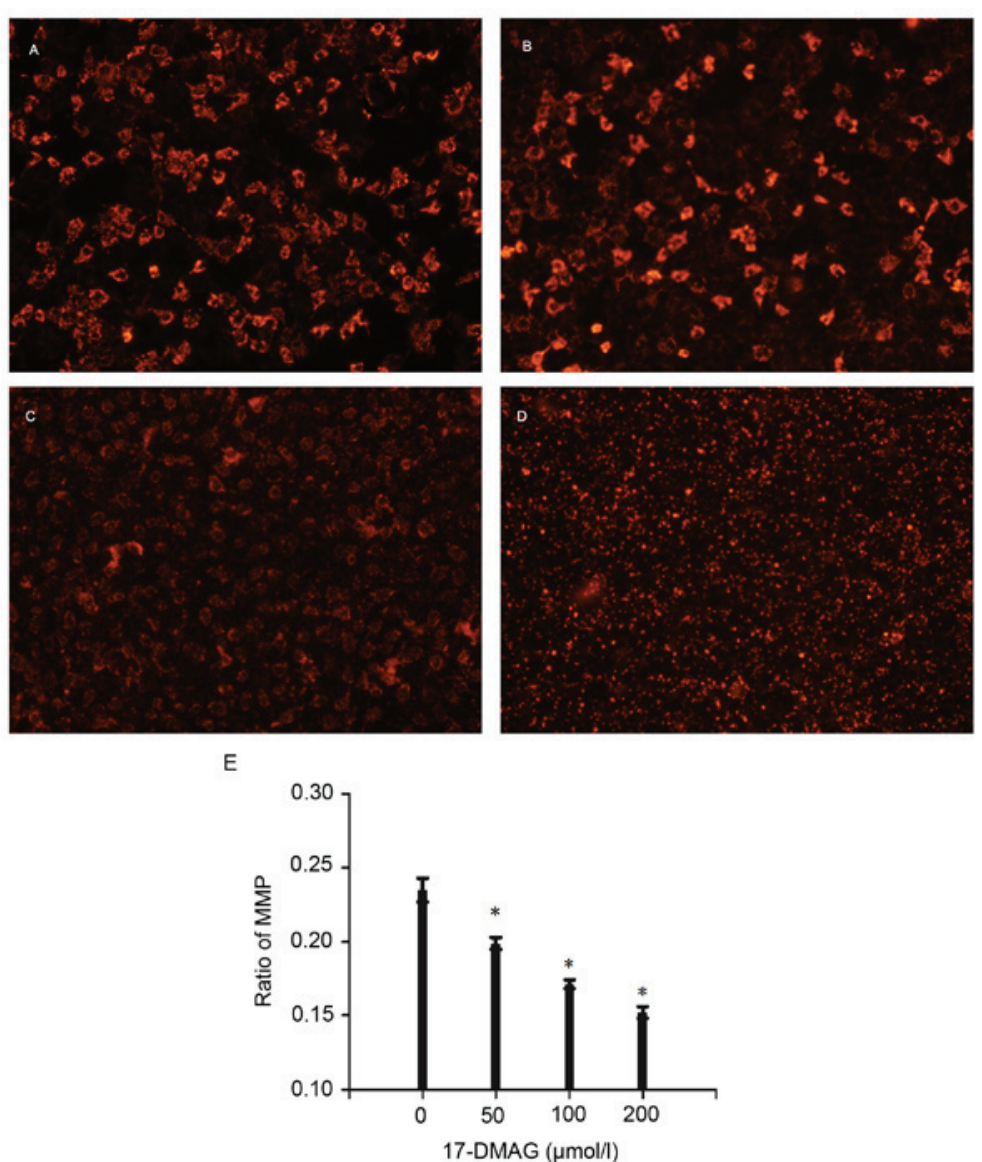

Figure 4. 17-DMAG reduces the mitochondrial membrane potential in SU-DHL-4 cells. Cells treated with (A) 0, (B) 50, (C) 100 and (D) $200 \mu$ mol/1 $17-D M A G$. Magnification, x100. (E) Quantification of the ratio of MMP. "P<0.05 vs. $0 \mu \mathrm{mol} / 1$. 17-DMAG, 17-dimethylaminoethylamino-17-demethoxy geldanamycin.

cultured with 17-DMAG at different concentrations for $24 \mathrm{~h}$ and stained with PI, following which the proportion of PI positive cells was measured using flow cytometry. The results revealed that the apoptosis of SU-DHL-4 cells was induced by 17-DMAG in a dose-dependent manner (Fig. 2 ; P $<0.05$ vs. $0 \mu \mathrm{mol} / \mathrm{l}$ ).

Cell apoptosis is induced by 17-DMAG via the mitochondrial pathway. In order to determine whether 17-DMAG induces apoptosis through the activation of the mitochondrial apoptotic pathway, the expression levels of several proteins involved in the mitochondrial apoptotic pathway were measured using western blot analysis. The levels of Bcl-2 family members were detected following treatment with 17-DMAG for $24 \mathrm{~h}$. The expression levels of anti-apoptotic proteins Bcl-2 were increased and HSPA5 were reduced following treatment with 17-DMAG, whereas levels of the proapoptotic protein Bax were constant (Fig. 3). Taken together, these results suggest that 17-DMAG induces apoptosis in DLBCL cells via the mitochondrial apoptotic pathway.

17-DMAG alleviates changes in MMP. MMP is an important determinant of early apoptosis (14). Accordingly, MMP was measured in DLBCL cells using JC-1 staining. In living DLBCL cells, JC-1 aggregates in the mitochondria and emits red fluorescence, whereas, in apoptotic DLBCL cells, JC-1 is a green fluorescence monomer and accumulates in the cytosol (15). The ratio of red to green fluorescence reflects the intensity of
MMP. In the control group, cells appeared orange-red (Fig. 4). In comparison, green fluorescence was more intense and red fluorescence was decreased in cells treated with 17-DMAG, indicating a decrease in the MMP. In the presence of 17-DMAG, the green fluorescent intensity was decreased, whereas the red fluorescence was increased. These results suggest that the 17-DMAG neuronal protective effect against cell apoptosis is achieved via the mitochondrial pathway.

\section{Discussion}

In the search for a novel, effective and safe pharmacological agent of natural origin for the treatment of cancer, the effect of 17-DMAG extract on apoptosis in SU-DHL-4 DLBCL cells was assessed. The results indicated that 17-DMAG exerts antiproliferative and proapoptotic effects on SU-DHL-4 cells via decreasing cell viability in a dose-dependent manner. The intrinsic or mitochondrial pathway is recognized as one of the major apoptosis signaling pathways. Specifically, the intrinsic pathway comprises mitochondria-dependent processes that result in decreasing Bcl-2 levels and activation of Bax (16). The present study focused on the molecular changes that occur early in the intrinsic apoptotic pathway, and it was observed that 17-DMAG causes a rapid decrease in the MMP.

Mitochondria serves an essential role in the intrinsic apoptotic pathway, which is mediated through amplification factors, including second mitochondria-derived activator of caspase, 
cytochrome $c$ and apoptotic inducer factors, and the execution of this pathway is achieved hby regulating the Bcl-2 family of proteins (17). The proapoptotic members, including Bax and $\mathrm{Bcl}-2$ homologous antagonist killer, and their antiapoptotic counterparts, including Bcl-2 and Bcl-2-like 1, typically maintain the balance (18). Bax translocates to the mitochondria and triggers the mitochondrial outer membrane potential, which facilitates the downstream apoptotic signaling assembly of pro-apoptotic factors in the apoptotic signal transduction pathways (19). In contrast, Bcl-2 overexpression blocks Bax from the mitochondria, thus inhibiting apoptosis (20). This is consistent with the results of the present study. 17-DMAG-induced apoptosis in SU-DHL-4 cells is associated with the downregulation of the BCL2 gene, whereas the upregulation of Bax gene expression indicates the involvement of the MMP.

The endoplasmic reticulum (ER) stress response has been demonstrated to be important for the growth and development of tumors under stressful growth conditions, including hypoxia and glucose deprivation, which are commonly encountered by the majority solid tumors (21). HSPA5 is a major ER stress-regulated protein that is involved in ER stress-induced apoptosis (22).

HSPA5 is a major regulator of the unfolded protein response and an ER-resident chaperone. Increasing evidence has indicated that HSPA5 is highly expressed in many tumor cell lines, and contributes to the invasion and metastasis of various human tumors (23). In addition, HSPA5 downregulation can lead to the detection of the ER stress-inducing anticancer therapies (21). The results of the present study indicated that the downregulation of HSP5A in SU-DHL-4 cells affects cell viability, whereas the activation of Bax abolishes the expression of Bcl-2. Previous studies have revealed that HSPA5 directly regulates the expression of target genes that lead to cell death or apoptosis in a p53-dependent or independent manner in cancer cells under ER stress $(23,24)$. The present study demonstrated that 17-DMAG activates Bax and downregulates the expression of Bcl-2 and HSPA5 in SU-DHL-4 cells. The effect of 17-DMAG may be achieved via a reduction in the activity of Bcl-2 and the maintenance of activity of Bax. During the MMP, the suppression of HSPA5 expression may inhibit ER stress. The results of the present study indicated that 17-DMAG serves a role in SU-DHL-4 cell apoptosis.

In conclusion, 17-DMAG induces apoptosis via the MMP pathway and blocks the ER stress pathway. Therefore, 17-DMAG may have potential as an effective therapeutic agent for DLBCL, although further study is required.

\section{References}

1. Nedomova R, Papajik T, Prochazka V, Indrak K and Jarosova M Cytogenetics and molecular cytogenetics in diffuse large B-cell lymphoma (DLBCL). Biomed Pap Med Fac Univ Palacky Olomouc Czech Repub 157: 239-247, 2013.

2. Abid MB, Nasim F, Anwar K and Pervez S: Diffuse large B cell lymphoma (DLBCL) in Pakistan: An emerging epidemic? Asian Pac J Cancer Prev 6: 531-534, 2005.
3. Sabattini E, Bacci F, Sagramoso C and Pileri SA: WHO classification of tumours of haematopoietic and lymphoid tissues in 2008: An overview. Pathologica 102: 83-87, 2010.

4. Stefancikova L, Moulis M, Fabian P, Vasova I, Zedek F, Ravcukova B, Muzik J, Kuglik P, Vranova V, Falkova I, et al: Prognostic impact of p53 aberrations for R-CHOP-treated patients with diffuse large B-cell lymphoma. Int J Oncol 39: 1413-1420, 2011.

5. Alzouebi M, Goepel JR, Horsman JM and Hancock BW: Primary thyroid lymphoma: The 40 year experience of a UK lymphoma treatment centre. Int J Oncol 40: 2075-2080, 2012.

6. Ahn JC, Kang JW, Shin JI and Chung PS: Combination treatment with photodynamic therapy and curcumin induces mitochondria-dependent apoptosis in AMC-HN3 cells. Int J Oncol 41: 2184-2190, 2012.

7. Prodromou C, Roe SM, O'Brien R, Ladbury JE, Piper PW and Pearl LH: Identification and structural characterization of the ATP/ADP-binding site in the Hsp90 molecular chaperone. Cell 90: 65-75, 1997.

8. da Silva VC and Ramos CH: The network interaction of the human cytosolic $90 \mathrm{kDa}$ heat shock protein Hsp90: A target for cancer therapeutics. J Proteomics 75: 2790-2802, 2012.

9. Taipale M, Jarosz DF and Lindquist S: HSP90 at the hub of protein homeostasis: Emerging mechanistic insights. Nat Rev Mol Cell Biol 11: 515-528, 2010.

10. Theodoraki MA and Caplan AJ: Quality control and fate determination of Hsp90 client proteins. Biochim Biophys Acta 1823: 683-688, 2012

11. Holzbeierlein JM, Windsperger A and Vielhauer G: Hsp90: A drug target? Curr Oncol Rep 12: 95-101, 2010.

12. Pacey S, Banerji U, Judson I and Workman P: Hsp 90 inhibitors in the clinic. Handb Exp Pharmacol: 331-358, 2006.

13. Neckers L and Ivy SP: Heat shock protein 90. Curr Opin Oncol 15: 419-424, 2003.

14. Colombini M: Ceramide channels and their role in mitochondria-mediated apoptosis. Biochim Biophys Acta 1797: 1239-1244, 2010.

15. Henry-Mowatt J, Dive C, Martinou JC and James D: Role of mitochondrial membrane permeabilization in apoptosis and cancer. Oncogene 23: 2850-2860, 2004.

16. McCarthy A, Marzec J, Clear A, Petty RD, Coutinho R, Matthews J, Wilson A, Iqbal S, Calaminici M, Gribben JG and Jia L: Dysregulation of autophagy in human follicular lymphoma is independent of overexpression of BCL-2. Oncotarget 5: 11653-11668, 2014

17. Kluck RM, Bossy-Wetzel E, Green DR and Newmeyer DD: The release of cytochrome $c$ from mitochondria: A primary site for Bcl-2 regulation of apoptosis. Science 275: 1132-1136, 1997.

18. Kang J, Chong SJ, Ooi VZ, Vali S, Kumar A, Kapoor S, Abbasi T, Hirpara JL, Loh T, Goh BC and Pervaiz S: Overexpression of Bcl-2 induces STAT-3 activation via an increase in mitochondrial superoxide. Oncotarget 6: 34191-34205, 2015.

19. Ghibelli L and Diederich M: Multistep and multitask Bax activation. Mitochondrion 10: 604-613, 2010.

20. Estaquier J, Vallette F, Vayssiere JL and Mignotte B: The mitochondrial pathways of apoptosis. Adv Exp Med Biol 942: 157-183, 2012.

21. Healy SJ, Gorman AM, Mousavi-Shafaei P, Gupta S and Samali A: Targeting the endoplasmic reticulum-stress response as an anticancer strategy. Eur J Pharmacol 625: 234-246, 2009.

22. Firczuk M, Gabrysiak M, Barankiewicz J, Domagala A, Nowis D, Kujawa M, Jankowska-Steifer E, Wachowska M, Glodkowska-Mrowka E, Korsak B, et al: GRP78-targeting subtilase cytotoxin sensitizes cancer cells to photodynamic therapy. Cell Death Dis 4: e741,2013.

23. Roller C and Maddalo D: The molecular chaperone GRP78/BiP in the development of chemoresistance: Mechanism and possible treatment. Front Pharmacol 4: 10, 2013.

24. Li W, Wang W, Dong H, Li Y, Li L, Han L, Han Z, Wang S, Ma D and Wang H: Cisplatin-induced senescence in ovarian cancer cells is mediated by GRP78. Oncol Rep 31: 2525-2534, 2014. 